Original Article

\title{
Our Experience of Posterior Fossa Tumors Surgeries
}

\author{
Tariq Imran Khokhar, Ajlan Ali, Ahmad Faizan Bukhari, Muhammad Naveed Majeed \\ Hafiz Abdul Majid, Asif Bashir
}

\begin{abstract}
Objective: In Neurosurgery Unit III, Punjab Institute of Neurosciences, Lahore, we evaluated our posterior fossa tumor surgery results, complications, and surgical outcomes.

Materials and Methods: Between January 2017 and September 2021, 80 patients with posterior fossa tumors who underwent surgical excision at the Neurosurgery Department-III of the Punjab Institute of Neurosciences in Lahore were studied retrospectively. For each patient, the diagnosis was made clinically and confirmed radiologically and histopathologically.
\end{abstract}

Results: Males comprised 47 percent (37) of the 80 cases, while females made up 53 percent (43). The average age was 15 (with a range of $6-30$ years). Medulloblastomas were the most frequent pathology in 25 patients (31\%), followed by ependymomas in 21 patients (26\%), pilocytic astrocytomas in 19 patients (24\%), and hemangioblastomas in 7 individuals (8.8\%). There were four cases of metastatic brain cancers (5\%), two cases of choroid plexus papilloma (2.5\%), one case of ganglioglioma (1.3\%), and one case of Dermoid cyst (1.3\%). In 90 percent of the cases (72 cases), gross total resection was obtained, while subtotal excision was performed in $10 \%$ of the cases ( 8 cases). The best results were seen in pilocytic astrocytoma surgery, followed by ependymoma surgery, whereas the worst results were seen in medulloblastoma surgery.

Conclusion: The surgical treatment of posterior fossa tumors still poses a significant challenge to neurosurgeons. Our experience shows that accepted results, complications, and surgical outcomes can be obtained by meticulous surgical techniques from previous clinical studies.

Keywords: Posterior Fossa; Medulloblastoma; Ependymoma.

Corresponding Author: Tariq Imran Khokhar

Department of Neurosurgery Unit III

Punjab Institute of Neurosciences (PINS), Lahore

Email: nsdrkhokhar2014@gmail.com

Date of Submission: 03-12-2021

Date of Revision: 25-12-2021

Date of Acceptance: 30-12-2021

Date of Online Publishing: 31-12-2021

Date of Print: 31-12-2021

DOI: $10.36552 /$ pjns.v25i4.619

\section{INTRODUCTION}

Tumors of the posterior fossa are one of the most common sites of malignancy in both adults and children. In children, the posterior fossa is home to more than half of all brain cancers, whereas, in adults, the posterior fossa is home to just 15 $20 \%$ of malignancies. ${ }^{1}$ They are the most dangerous tumors, with a high risk of morbidity and mortality, particularly among children. The cerebellum, brainstem, or fourth ventricle may all be affected by tumors in this area. 
Medulloblastoma, ependymoma, and pilocytic astrocytoma are the most prevalent tumors in children. The posterior fossa is the most prevalent site of metastasis and hemangioblastoma and lymphoma in adults. ${ }^{2}$ The size, location, biological behavior, and aggressiveness of these tumors all influence their clinical presentation. ${ }^{3}$ Due to focused compression on the cerebellum and brainstem, the majority of patients present with symptoms of elevated ICP, such as headache, vomiting, and vertigo. ${ }^{4}$ The diagnostic method of choice is MRI brain with contrast. In most cases, an MRI of the spine should be performed to check for any dropmets. The preferred therapeutic method is gross complete resection. ${ }^{5}$ The amount of tumor excision is the single most critical determinant in determining the survival of individuals with posterior fossa cancers. As a result, the operating surgeon must achieve the maximum safe resection, as the extent of resection is critical not only for survival but also for survival quality. ${ }^{6}$ It's a surgical conundrum that as the extent of resection is increased, so does the risk of morbidity and mortality. Patients with posterior fossa tumors have surgery to decompress the posterior fossa, obtain tumor type using histology, and choose the best course of treatment based on the tumor type. Cushing was the first to publish a case series of 61 posterior fossa tumor patients. He revealed that the majority of medulloblastomas result in death. ${ }^{7}$

Despite advances in micro-neurosurgery procedures, posterior fossa tumor surgery is still associated with high rates of death in our region of the world, particularly among children. The purpose of this study is to share our anecdotal experience with posterior fossa tumor surgeries and to look at the results in terms of CSF leaking, post-operative infections including meningitis, hydrocephalus necessitating a VP shunt, and mortality.

\section{MATERIALS AND METHODS}

\section{Study Design}

From the 1st of January 2017 to the 30th of September 2021, this study was conducted in the department of Neurosurgery Unit - III. This study included all male and female patients aged 6 to 30 who underwent posterior fossa tumor surgery.

\section{Inclusion Criteria}

This study included all male and female patients aged 6 to 30 who underwent posterior fossa tumor surgery.

\section{Exclusion Criteria}

The study excluded all procedures on the Cerebellopontine Angle.

\section{Operative Technique}

All of the patients had undergone a thorough clinical assessment by a consultant neurosurgeon, as well as a radiological examination that included a CT brain plain scan and an MRI brain with contrast. The same surgical team operated on all of the patients. The patient was positioned prone on the table with support for the thorax, pelvis, and legs during surgery. The belly should be free from this support. In some patients, the head was clamped in a Mayfield clamp, and in others, it was mounted on Horseshoe. To get a better view of the lower occiput and neck, the head was flexed more. Extending from external occipital protuberance to the posterior arch of $\mathrm{C} 1$, a midline skin incision was made. Because subsequent surgery and radiotherapy may result in increased cervical instability, the spinous process of C2 was not exposed to retain its ligamentous and muscular attachment. In all patients, a sub-occipital craniectomy was performed. We removed the posterior arch of C1 in the majority of cases because the tumor was expanding below the foramen magnum. A "Y" shaped dural opening was performed over both 
hemispheres, with the "long leg" of the $Y$ in the midline and the occipital sinus ligated in the midline. After reflection of the dura and the opening of the cisterna magna to drain CSF, the microscope was brought into the field. The telovaler method was used to treat malignancies in the fourth ventricle. Dissection began on one side, medial to the tonsil, between the tonsil and the uvula, using the telovaler approach. To avoid any neurological deficit, the tumor bulk was gradually resected, and any tumor on the floor of the fourth ventricles was decreased as much as possible without disrupting the floor.

For cancers in the cerebellar hemispheres, a trans-cortical approach was used, with gross complete excision of the tumor and, in the event of cystic tumors, resection of the capsule as well. Hemostasis was achieved using sponge stone and surgicels, and diathermy coagulation was minimized as much as feasible. If there was tightness or compression on the cerebellar hemispheres, the dura was closed as watertight as feasible without producing compression, or a duro-plasty was performed. The placement of a ventriculoperitoneal shunt was done urgently in some patients before tumor removal if they had an acute disturbance of consciousness, and in a few individuals following surgery. Specimens were sent for histological investigation after surgery. All patients were admitted to the intensive care unit immediately after surgery, and the conscious state was closely monitored. One week and one month after surgery, clinical and radiological follow-up was performed.

\section{Data analysis}

SPSS version 22 was used for statistical analysis. The average and mean standard deviations were used to present the data.

\section{RESULTS}

\section{Age and Gender Distribution}

From January 1, 2017, to September 30, 2021, 80 patients with posterior fossa tumors were operated on at Neurosurgery Unit-III, Punjab Institute of Neurosciences, Lahore, Pakistan. The participants ranged in age from 6 to 30, with an average of 15 years. Males made up 37 (47\%) patients, while females made up 43 (53\%).

\section{Tumor Resection}

72 patients (90\%) got microscopic gross complete resection, while 8 patients (10\%) underwent subtotal resection as mentioned in Table 2.

\section{Tumor Resection}

In 72 patients (90 percent), microscopic gross total resection was obtained, whereas subtotal resection was performed in eight cases (10 percent) as mentioned in Table 2. These patients received no adjuvant therapy. All 25 medulloblastoma patients were referred to an outpatient oncology center for chemo and radiotherapy. Two patients with medulloblastoma, one patient with ependymoma, and one patient with pilocytic astrocytoma had tumor recurrence.

In this study, great surgical outcomes were seen in 22 instances (28\%) and good outcomes in 47 cases (59\%) while poor outcomes were seen in 11 cases (13 percent). Pilocytic astrocytoma surgery had the best outcome, followed by ependymoma surgery, whereas medulloblastoma and metastatic tumors had the worst outcome, which was statistically insignificant. All the images are shared in results after the patient's consent.

\section{Histopathology}

The following histopathological diagnoses were found in the specimens as mentioned in Table $\mathbf{3}$ : medulloblastoma in 25 patients (31\%); ependymoma in 21 patients (26\%); pilocytic 
astrocytoma in 19 patients (24\%); and hemangioblastoma in 7 patients (8.8 percent). There were four cases of metastatic brain cancers (5\%), two cases of choroid plexus papilloma (2.5\%), one case of ganglioglioma (1.3\%), and one case of Dermoid cyst (1.3\%).

Table 1: Clinical Presentation.

\begin{tabular}{|lcc|} 
Clinical Presentation & No. of Patients & Percentage \\
Headache & 74 & 92 \\
Vomiting & 60 & 75 \\
Ataxia & 22 & 27 \\
CN Palsy & 16 & 20 \\
\hline
\end{tabular}

Table 2: Tumor Resection.

\begin{tabular}{lcc} 
Parameters & No. of Patients & Percentage \\
Gross Total & 72 & $90 \%$ \\
Sub-Total & 8 & $10.00 \%$ \\
\hline
\end{tabular}

Table 3: Histopathology.

\begin{tabular}{lcc|} 
Parameters & No. of Patients & Percentage \\
Medulloblastoma & 25 & $31 \%$ \\
Ependymoma & 21 & $26 \%$ \\
Pilocytic Astrocytoma & 19 & $24 \%$ \\
Hemangioblastoma & 7 & $9 \%$ \\
Metastatic & 4 & $5 \%$ \\
Choroid P. Papilloma & 2 & $3 \%$ \\
Ganglioglioma & 1 & $1 \%$ \\
Dermoid Cyst & 1 & $1 \%$ \\
Total & $\mathbf{8 0}$ & $\mathbf{1 0 0 \%}$ \\
\hline
\end{tabular}

Table 4: Complications.

\begin{tabular}{lcc} 
Complications & No. of Patients & Percentage \\
CSF Leak & 6 & $8 \%$ \\
Meningitis & 2 & $3 \%$ \\
Wound Infection & 4 & $5 \%$ \\
CN palsy & 3 & $4 \%$ \\
Cerebellar Hematoma & 4 & $5 \%$ \\
Hydrocephalous & 21 & $26 \%$ \\
Requiring VP Shunt & 2 & $2.6 \%$ \\
Mortality & 2 & \\
\hline
\end{tabular}

\section{Complications}

Wound infection in 6 (8\%), meningitis in 2 (3\%),
CSF leak in 6 (8\%), and Cranial nerve palsies in 3 (4\%), cerebellar hematoma in 4 and (5\%), hydrocephalus requiring VP shunt in 21 (26\%) patients were all complications noted as mentioned in Table $\mathbf{4}$ andconnected with surgical sites that were largely treated with medicinal or surgical care and did not lead to death.

\section{DISCUSSION}

Because of the complicated neurovascular structures present in this area, malignancies in the posterior fossa are difficult to operate on. Cerebellar, intraventricular, and brainstem tumors are the three types of tumors that can be classified based on their location. Cerebellar cancers are near to the brainstem, and the exophytic component of a brainstem tumor can grow posteriorly toward the fourth ventricle and cerebellum. ${ }^{8}$ As a result, cerebellar signs and symptoms such as vertigo, nausea, vomiting, ataxia, and nystagmus may be present. Cerebellar tumors and intraventricular tumors that enter the brainstem, on the other hand, cause symptoms such as cranial nerve palsies and motor impairments. $^{9}$ As a result, simply based on symptomatology, distinguishing between brainstem tumors and cerebellar tumors is challenging.

Our case series includes individuals of all ages, the majority of whom are young adults. Our population was overwhelmingly female. This contrasts with a similar study by Jahanzaibkakar et al., which found a male-dominated population. ${ }^{10}$ In our case study, the most prevalent clinical manifestation was headache and vomiting, which has also been documented in other case studies. Emara et al. reported 90.9 percent of cases presenting with headache in their case series, which is very comparable to our results (90 percent)as mentioned in Table $1 .{ }^{11}$ In $90 \%$ of the tumors, we were able to do gross total resection; however, in $10 \%$ of the tumors when subtotal resection was performed, the tumor was 
grossly adherent to the brainstem. As a result, that area of the tumor was not removed and was left as is to avoid any damage to important tissuesas mentioned in Table 2. For radiotherapy and chemotherapy, all of these patients were referred to oncology. KamilMelihakay et al. reported a gross total excision rate of 71.4 percent in research. ${ }^{12}$ The most prevalent histological diagnosis was medulloblastoma (31\%), followed by ependymoma (26\%), Pilocytic astrocytoma (25\%), Hemangioblastom (8.8\%), Metastatic (5\%), Choroid Plexus Papilloma (2.5\%), and Ganglioglioma (2.5\%). (1 percent )as mentioned in Table 3. In their investigation, Ali R Hamdan et al found that medulloblastoma was the most common kind of tumor present (40\%); however, they did not find any cases of ependymoma, and their histological diagnostic of brain Mets was comparably higher (7\%) than our case series. ${ }^{13}$ The most prevalent tumor in the adult population in the posterior fossa is Mets, according to the literature, however, our investigation found different. In terms of biological behavior, medulloblastoma is an aggressive tumor.Due to tumor recurrence, two patients had to have a second operation in less than a year. The majority of the patients in our case series showed increased ICP symptoms of headache and vomiting when we operated on them. Ten of the 23 patients who needed ventriculoperitoneal (VP) shunting had it done before the tumor was removed, and 13 had it done after. According to Robert et al's study, 33.3 percent of patients needed a VP shunt, which is similar to our findings. ${ }^{14}$

Sawayar et al. divided cranial neurotomy problems into three categories: neurological, regional, and systemic issues. ${ }^{15}$ Surgical operations are linked to neurological and regional problems. As a result, we concentrated our research on these two issues. One of the most common problems following skull base surgery is the leakage of cerebrospinal fluid (CSF). Fluid can leak from the nose, ear, or a cut on rare occasions. We experienced CSF leaking in six cases; two of them were handled with reenforcement stitches, while the other four required VP shunting due to the presence of

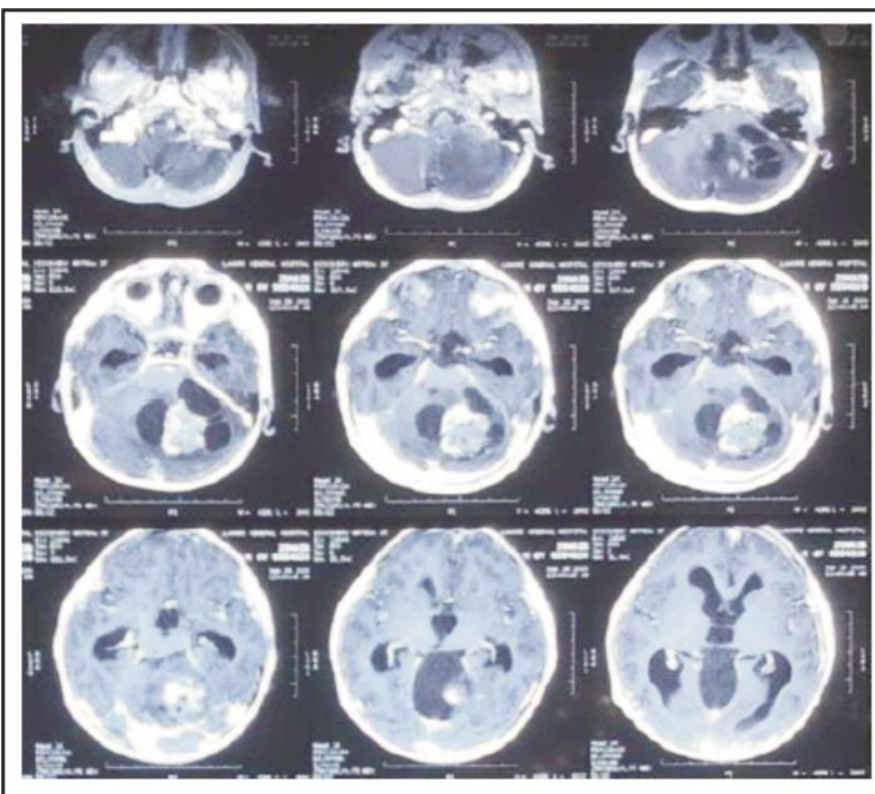

(a)

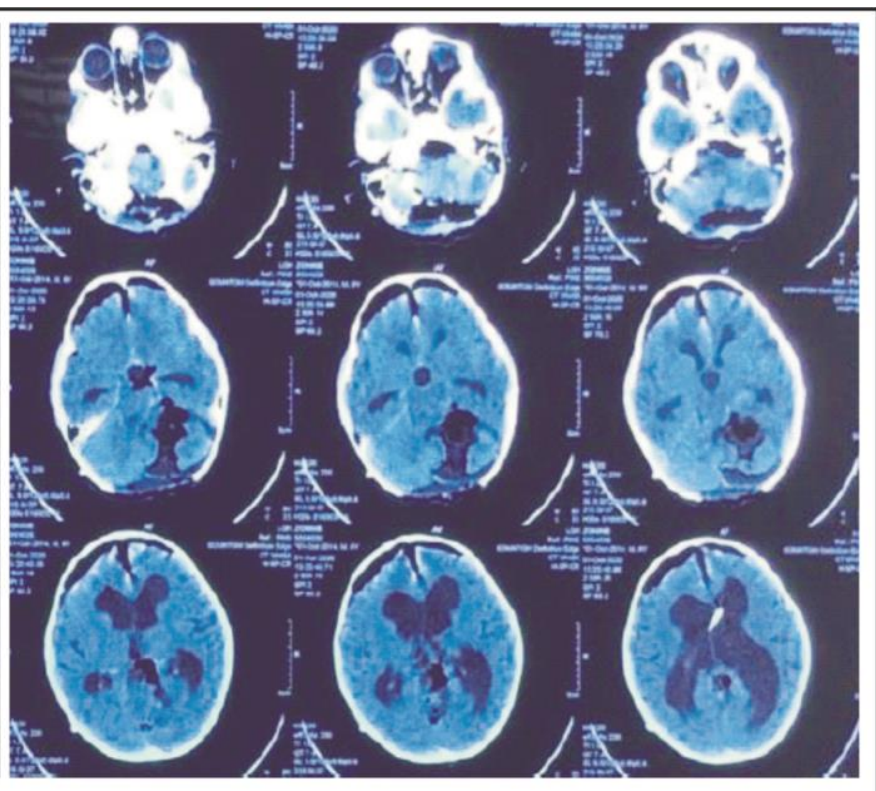

(b)

Figure 1: An axial segment of T1 weighted contrast MRI of a patient revealing a midline Solid-cystic cerebellar tumor contrastenhancing nodular lesion (a) postoperative CT scan demonstrating no residual tumor (b). 


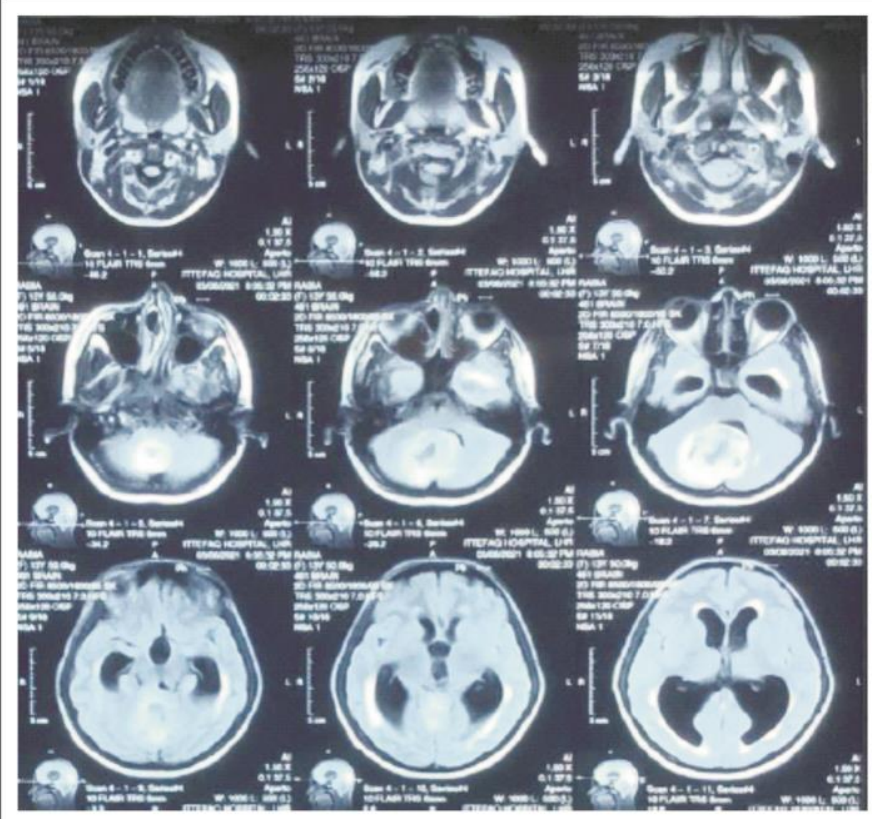

(a)

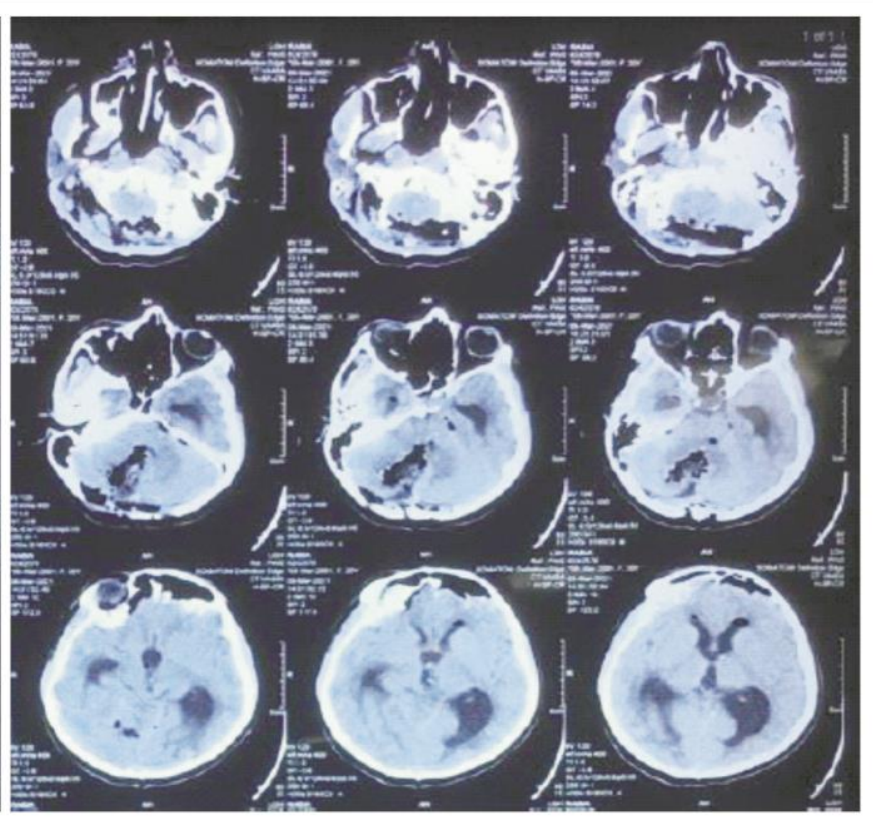

(b)

Figure 2: An axial segment of $\mathrm{T} 1$ weighted Contrast MRI of a patient with a midline solid cystic contrast-enhancing mass lesion (medulloblastoma) (a) postoperative CT scan demonstrating no residual tumor (b).

hydrocephalus in all of the patients.CSF leakage is most commonly caused by faulty dural closures, hence dural closures should always be watertight. In their series, Saway et al reported roughly 26\% of cases with CSF leakage, which is extremely significant when compared to our study, which reported just $8 \%$ of cases with CSF leakage. ${ }^{16}$ Three patients had an intracerebellar hemorrhage right after surgery, while one patient had a delayed intracerebellar hematoma. All of these cases were treated with re-exploration and hematoma evacuation.Wound infection occurred in $5 \%$ of our subjects after surgery in our study. When compared to research by ArvindDubey et al., there were 13.6 percent cases of wound infection. Persistent CSF leaks, foreign bodies, lengthy surgery, long-term steroid usage, and diabetes have all been linked to an increased risk of infection. ${ }^{17}$

In our study series, the total mortality rate was $2 \%$ as mentioned in Table 4 . The patient's age, comorbidities, clinical characteristics of the tumor, and neurological impairment all raise the risk of death. The most important factors in postoperative death were postoperative hematoma, edema with herniation, and vascular compromise with consequent infarction. The remaining deaths were caused by aspiration pneumonia caused by cranial nerve impairments, as well as various medical diseases such as pulmonary embolism, myocardial infarction, and sepsis. Wound infection, meningitis, CSF leak, and Cranial nerve palsies were all complications connected with surgical sites that were largely treated with medicinal or surgical care and did not lead to death. Even though they are histologically benign tumors, ependymomas are the hardest to cure. Early childhood is when they are more common. After surgical excision, the rate of recurrence is extremely high. Microsurgical resection was conducted through painstaking dissection to avoid probable neurological impairments and achieve gross complete resection. In cancers that had subtotal resection or had a high recurrence rate, postoperative radiation and chemotherapy were used as an 
adjuvant. Patients who had radiotherapy after surgery had better outcomes than those who only received surgery. In four years, we operated on 21 patients with ependymoma, and two of them (10\%) died. As a supplement, all of these patients received postoperative radiation.

\section{CONCLUSION}

When compared to malignancies of the supratentorial compartment, microsurgery of the posterior fossa has a much higher risk of complications. In this case series, we discovered that posterior fossa tumor surgery may be conducted safely in the majority of patients with a low risk of morbidity and mortality. To avoid any unforeseen complications, careful perioperative planning, rigorous microsurgical dissection, good wound closure, and strict adherence to proper aseptic methods are required. Furthermore, detecting surgical problems early and providing adequate therapy can help to prevent lifelong disability. In comparison to existing published material from our region of the world, our experience illustrates the acceptable results, complications, and surgical outcomes in posterior fossa tumor removal.

\section{REFERENCES}

1. Vernon-Levett P, Geller M: Posterior fossa tumors in children: A case study. AACN Cl in Issues, 1997; 8: 214-226.

2. Ramanan $\mathrm{M}$, Chaseling R. Paediatric brain tumours treated at a single, tertiary paediatric neurosurgical referral centre from 1999 to 2010 in Australia. J Clin Neurosci. 2012; 19: 1387-91.

3. Witt $H$, Mack SC, Ryzhova $M$, Bender $S$, Sill $M$, Isserlin R, et al. Delineation of two clinically and molecularly distinct subgroups of posterior fossa ependymoma. Cancer Cell, 2011; 20: 143-57.

4. Mpairamidis E, Alexiou GA, Stefanaki K, Manolakos I, Sfakianos G, Prodromou N. Posterior fossa tumor in a 12 year-old boy. Brain Pathol. 2009; 19: 341-2.

5. MacDonald T. Medulloblastoma. eMedicine; 2009. Available from: http://emedicine.medscape.com/article/987886print. [Last Accessed on 2014 Nov 11].

6. Soffietti $R$, Baumert BG, Bello $L$, von Deimling A, Duffau $H$, Frénay $M$, et al. Guidelines on management of low - grade gliomas: Report of an EFNS-EANO task force. Eur J Neurol. 2010; 17: 1124-33.

7. Stupp $R$, Brada $M$, van den Bent MJ, Tonn JC, Pentheroudakis G. ESMO Guidelines Working Group. High-grade glioma: ESMO clinical practice guidelines for diagnosis, treatment and follow-up. Ann Oncol. 2014; 25 Suppl. 3: iii 93-101.

8. Wright KD, Gajjar A. Current treatment options for pediatric and adult patients with ependymoma. Curr Treat Options Oncol. 2012; 13: 465-77.

9. Steinbok $P$, Mangat JS, Kerr JM, Sargent $M$, Suryaningtyas $W$, Singhal $A$, et al. Neurological morbidity of surgical resection of pediatric cerebellar astrocytomas. Childs Nerv Syst. 2013; 29: 1269-75.

10. Kakar J, Ashraf J, Khan AA, Imran M, Rehmani MA, Ghori SA, Ali MF. The satisfactory surgical outcome of posterior fossa brain tumors in children at civil hospital, Karachi. Asian Journal of Neurosurgery, 2020 Apr; 15 (2): 377.

11. Ries LA, Smith MA, Gurney JG, Linet M, Tamra T, Young $J L$, et al., editors. Cancer Incidence and Survival Among Children and Adolescents: United States SEER Program, 1975 - 1995.

12. Akay KM, Izci Y, Baysefer A, Atabey C, Kısmet E, Timurkaynak E. Surgical outcomes of cerebellar tumors in children. Pediatric Neurosurgery, 2004; 40 (5): 220-5.

13. Essa $A E$, Hamdan $A R$. Posterior fossa intra-axial tumors: surgical outcomes. The Medical Journal of Cairo University, 2018 Dec. 1; 86 (December): 3433-9.

14. Shih RY, Smirniotopoulos JG. Posterior fossa tumors in adult patients. Neuroimaging Clinics, 2016 Nov. 1; 26 (4): 493-510.

15. Sawaya R, Hammoud M, Schoppa D, Hess KR, Wu SZ, Shi WM, Wildrick DM. Neurosurgical outcomes in a modern series of 400 craniotomies for treatment of parenchymal tumors. Neurosurgery, 1998 May 1; 42 (5): 1044-55.

16. ESSA AE, HAMDAN AR. Posterior fossa intra-axial tumors: surgical outcomes. The Medical Journal of Cairo University. 2018 Dec. 1; 86 (December): 
3433-9.

17. Dubey A, Sung WS, Shaya M, Patwardhan R, Willis B, Smith D, Nanda A. Complications of posterior cranial fossa surgery-an institutional experience of 500 patients. Surgical Neurology, 2009 Oct. 1; 72 (4): $369-75$.

\section{Additional Information}

Disclosures: Authors report no conflict of interest.

Ethical Review Board Approval: The study was conformed to the ethical review board requirements.

Human Subjects: Consent was obtained by all patients/participants in this study.

\section{Conflicts of Interest:}

In compliance with the ICMJE uniform disclosure form, all authors declare the following:

Financial Relationships: All authors have declared that they have no financial relationships at present or within the previous three years with any organizations that might have an interest in the submitted work.

Other Relationships: All authors have declared that there are no other relationships or activities that could appear to have influenced the submitted work.

\section{AUTHORS CONTRIBUTIONS}

\begin{tabular}{|c|c|c|}
\hline Sr.\# & Author's Full Name & Intellectual Contribution to Paper in Terms of: \\
\hline 1. & Tariq Imran Khokhar & $\begin{array}{l}\text { 1. Study Design and Methodology. Paper Writing, Referencing and } \\
\text { Data Calculations. }\end{array}$ \\
\hline 2. & Ajlan Ali & 2. Data Collection and Calculations. \\
\hline 3. & Ahmad Faizan Bukhari & 3. Analysis of Data and Interpretation of Results etc. \\
\hline 4. & Muhammad Naveed Majeed & 4. Literature Review and Manuscript Writing. \\
\hline 5. & Hafiz Abdul Majid & 5. Literature Review. \\
\hline 6. & Asif Bashir & 6. Quality Insurer. \\
\hline
\end{tabular}

